\title{
Spatially homogeneous ground state of the two-dimensional Hubbard model
}

\author{
Federico Becca, Massimo Capone, and Sandro Sorella \\ Istituto Nazionale per la Fisica della Materia and International School for Advanced Studies, Via Beirut 4, 34013 Trieste, Italy
}

(October 28, 2018)

\begin{abstract}
We investigate the stability with respect to phase separation or charge density-wave formation of the two-dimensional Hubbard model for various values of the local Coulomb repulsion and electron densities using Green-function Monte Carlo techniques. The well known sign problem is particularly serious in the relevant region of small hole doping. We show that the difference in accuracy for different doping makes it very difficult to probe the phase separation instability using only energy calculations, even in the weak-coupling limit $(U=4 t)$ where reliable results are available. By contrast, the knowledge of the charge correlation functions allows us to provide clear evidence of a spatially homogeneous ground state up to $U=10 t$.
\end{abstract}

71.10.Fd, 71.45.Lr, 74.20.-z

\section{INTRODUCTION}

Since the discovery of high-temperature superconductivity, the two-dimensional Hubbard modelt has been the subject of a huge amount of work. Indeed, it is widely accepted that, despite the complicate structure of these materials, a key role is played by the electron correlation in the $\mathrm{CuO}$ planes, well represented by the Hubbard model.

This model and the related $t-J$ model reproduce, at least qualitatively, some of the physical properties of cuprates. For example, at half-filling (i.e., when the number of electrons is equal to the number of sites) the ground state (GS) is an antiferromagnetic insulator, and upon doping the antiferromagnetism is strongly suppressed. The possible insurgence of superconductivity upon doping and the symmetry of the order parameter in the model are still open questions. Another important point to study is the stability with respect to phase separation (PS) or charge-density waves (CDW) near halffilling. Indeed, both PS and CDW hape been experimentall pobserved in different systems authors 4 have pointed out a possible relation between charge instability and superconductivity.

Because of the strongly interacting nature of these systems, important insights came from nonperturbative numerical methods.

The exact diagonalization methods, e.g., Lanczos, are strongly limited by the exponential growth of the Hilbert space, and in practice it is possible to diagonalize only up to about 20 sites. A remarkable development of the exact diagonalization strategy is the density matrix renormalization groupl, which allows us to compute the GS using an iteratively improved basis. Despite the accuracy of this technique for one-dimensional and quasi-onedimensional systems, there is no straightforward generalization for higher dimensions.

Attractive alternatives are stochastic methods such as Quantum Monte Carlo (QMC) that do not suffer from severe limitations in terms of lattice size and hence allow us to study fairly large systems. The simplest QMC simulation is the variational one, in which it is possible to compute the expectation values of the energy and all the correlation functions for a given wave function. The limitation of this method is that one has to guess a priori the form of the GS.

QMC methods allow us to sample directly the GS by using projection techniques, the simplest one being the so-called power method, namely the simple idea that an iterative application of the Hamiltonian $H$ filters out the ground state starting from an arbitrary state nonorthogonal to it. These approaches suffer, in fermionic systems, from the so-called sign problem. Indeed, due to the antisymmetry of the wave function under permutations of two particles, one gets after a few power iterations opposite contributions leading to the cancellation of large and fluctuating weights.

An alternative approach, which is quite efficient for the Hubbard model, is the auxiliary field quantum Monte Carlo (AFQMC) . The Coulomb interaction is linearized by using a Hubbard-Stratonovic transformation. In this way it is possible to apply exactly the operator $e^{-\beta H}$ to a Slater determinant. In practice, AFQMC is very accurate only for small values of the Coulomb repulsion, whereas, when the interaction strength becomes comparable with the bare bandwidth, large fluctuations make the simulations rather unstable and inefficient, because the sign problem becomes particularly severe for strong Coulomb repulsion.

A sophisticated implementation of the power method is the Green-function Monte Carlo (GFMC) applied to lattice model\$s. In principle, this method gives unbiased results but in practice, for fermion systems, the sign problem makes any simulation prohibitive, because of statistical errors. Recently, many attempts have been made to deal with fermions and overcome the sign problement. The fixed-node approximation 10 is introduced for the GFMC and replaces the original Hamiltonian with an effective one free of the sign problem, in which the nodes are fixed to be those of the so-called guiding wave function. In practice, the most common choice is the best variational wave function available, i.e., the one with the lowest energy. The fixed-node energy is an upper bound to the true GS energy and the method is variational. 
Moreover, it becomes exact if the guiding function is chosen to be the exact GS function. Although this approximation can be uncontrolled and needs an external input, it is quite accurate with respect to both energy and correlation functions for small sizes.

An alternative approach, recently proposed by one of us, is to use the GFMC with a stochastic reconfiguration (SR) 11 . The main idea of this method is to use a suitable reference dynamics free of the sign problem to constrain the true dynamics. The fixed-node and a variational dynamics are natural candidates as references. At each reconfiguration the amplitudes of the GS are chosen as small perturbations of the reference wave function amplitudes such that the mixed averages of a few physically relevant quantities are conserved. The method is exact if all the operators in the Hilbert space are reconfigured, but it has been shown that a major role is played by a very small number of operators 12

In this paper, we present a systematic study on the Hubbard model comparing different QMC techniques by studying in particular the stability of PS and CDW near half-filling for various values of the Coulomb repulsion. In Sec. II we introduce the Hubbard model and we discuss the implementation of our approximations, in Sec. III we report our results, and finally in Sec. IV] we give a brief summary.

\section{THE MODEL}

We consider the Hubbard model on a square lattice of $L$ sites with $N=N_{\uparrow}+N_{\downarrow}$ particles and $N_{\uparrow}=N_{\downarrow}$, where $N_{\uparrow}\left(N_{\downarrow}\right)$ is the number of spin-up (-down) particles. In order to study PS as close as possible to half-filling using only closed-shell configurations, we consider square lattices tilted by $45^{\circ}$ with $L^{2}=2 l^{2}$ and $l$ odd. In this way half-filling is a closed shell and the first doped closed shell has eight holes independent of $L$. The Hamiltonian reads

$$
H=-t \sum_{\langle i, j\rangle, \sigma} c_{i, \sigma}^{\dagger} c_{j, \sigma}+U \sum_{i} n_{i, \uparrow} n_{i, \downarrow}
$$

where \langle\rangle stands for nearest neighbors, $c_{i, \sigma}\left(c_{i, \sigma}^{\dagger}\right)$ destroys (creates) an electron with spin $\sigma$ at site $i$, and $n_{i, \sigma}=$ $c_{i, \sigma}^{\dagger} c_{i, \sigma}$. In the following, all energies are measured in units of $t$.

As already pointed out above, in the presence of the sign problem the choice of the guiding wave function is crucial. Our wave function reads

$$
\left|\Psi_{G}\right\rangle=\mathcal{P}_{S z=0} \mathcal{P}_{g} \mathcal{J}|\mathcal{D}\rangle,
$$

where $|\mathcal{D}\rangle$ is a Slater determinant in which the orbitals are suitably chosen (see below), $\mathcal{P}_{S z=0}$ is the projector onto the subspace with $N_{\uparrow}=N_{\downarrow}$, i.e., with zero total spin component along the $z$ axis, $\mathcal{P}_{g}$ is a Gutzwiller operator that inhibits the double occupancies $\mathcal{P}_{g}=$ $\exp \left(-g \sum_{i} n_{i \uparrow} n_{i \downarrow}\right)$, where $g$ is a variational parameter, and $\mathcal{J}$ is a Jastrow factor $\mathcal{J}=\exp \left(\frac{\gamma}{2} \sum_{i, j} v_{i, j} S_{i}^{z} S_{j}^{z}\right)$, where $\gamma$ is another variational parameter and $v_{i, j}$ is taken from spin-waves theory 13 . Care must be taken in the choice of the orbitals appearing in the Slater determinant. The most common choice is to take the orbitals from a Hartree-Fock (HF) approximation of the Hamiltonian breaking the $\mathrm{SU}(2)$ spin rotation symmetry along the $z$ axis.

$$
H=-t \sum_{\langle i, j\rangle, \sigma} c_{i, \sigma}^{\dagger} c_{j, \sigma}+\frac{U}{2} \sum_{i, \sigma}\left[\left\langle n_{i}\right\rangle-\sigma(-1)^{R_{i}}\left\langle m_{i}\right\rangle\right] n_{i, \sigma},
$$

where

$$
\begin{aligned}
\left\langle n_{i}\right\rangle & =\left\langle n_{i, \uparrow}\right\rangle+\left\langle n_{i, \downarrow}\right\rangle, \\
\left\langle m_{i}\right\rangle & =(-1)^{R_{i}}\left[\left\langle n_{i, \uparrow}\right\rangle-\left\langle n_{i, \downarrow}\right\rangle\right] .
\end{aligned}
$$

We consider only fillings which are closed shells for $U=0$ and where a solution with constant density $\left\langle n_{i}\right\rangle=\frac{N}{L}=n$ and staggered magnetization $\left\langle m_{i}\right\rangle=m$ are found. In this case, the HF many-body wave function can be written as

$$
|\mathcal{D}\rangle=\prod_{k}^{1, \ldots, N_{\uparrow}} \beta_{k, \uparrow}^{\dagger} \prod_{q}^{1, \ldots, N_{\downarrow}} \beta_{q, \downarrow}^{\dagger}|0\rangle,
$$

where the quasiparticles have definite momentum modulo $Q=(\pi, \pi)$ and definite spin, since the antiferromagnetic order parameter is along the $z$ axis

$$
\beta_{k, \sigma}^{\dagger}=u_{k} c_{k, \sigma}^{\dagger}+\sigma v_{k} c_{k+Q, \sigma}^{\dagger},
$$

$k$ is in the reduced magnetic Brillouin zone, and $u_{k}$ and $v_{k}$ are defined in Ref14. It is worth noting that for $U / t \rightarrow$ $\infty, u_{k}=v_{k}=\frac{1}{\sqrt{2}}$, namely the spin up and the spin down are in different sub-lattices (classical Néel state).

In a previous work 15 the wave function (2) with $|\mathcal{D}\rangle$ given by Eq. (6) has been found to be a rather poor approximation for large $U / t$ at half-filling. In particular, in this representation the Jastrow factor $\mathcal{J}$ does not play any important role.

We propose a wave function which is a straightforward generalization of the one successfully used for the Heisenberg model16. The fundamental ingredient is to allow spin fluctuations perpendicular to the staggered magnetization. An easy implementation of this idea is to put the magnetization in the $x-y$ plane allowing transverse fluctuations along the $z$ axis through a Jastrow-like factor 13 . This is achieved by a $\frac{\pi}{2}$ rotation $U_{y}\left(\frac{\pi}{2}\right)$ around the $y$ axis of the canonical operators:

$$
\begin{aligned}
& U_{y}^{\dagger}\left(\frac{\pi}{2}\right) c_{i, \uparrow}^{\dagger} U_{y}\left(\frac{\pi}{2}\right)=\frac{1}{\sqrt{2}}\left(c_{i, \uparrow}^{\dagger}+c_{i, \downarrow}^{\dagger}\right), \\
& U_{y}^{\dagger}\left(\frac{\pi}{2}\right) c_{i, \downarrow}^{\dagger} U_{y}\left(\frac{\pi}{2}\right)=\frac{1}{\sqrt{2}}\left(c_{i, \uparrow}^{\dagger}-c_{i, \downarrow}^{\dagger}\right) .
\end{aligned}
$$


The fermionic part of our guiding wave function is therefore defined as a Slater determinant of the transformed orbitals,

$$
\begin{aligned}
& \beta_{k,+}^{\dagger}=U_{y}^{\dagger}\left(\frac{\pi}{2}\right) \beta_{k, \uparrow}^{\dagger} U_{y}\left(\frac{\pi}{2}\right), \\
& \beta_{k,-}^{\dagger}=U_{y}^{\dagger}\left(\frac{\pi}{2}\right) \beta_{k, \downarrow}^{\dagger} U_{y}\left(\frac{\pi}{2}\right),
\end{aligned}
$$

namely it is given by

$$
|\mathcal{D}\rangle=\prod_{k}^{1, \ldots, N_{\uparrow}} \beta_{k,+}^{\dagger} \prod_{q}^{1, \ldots, N_{\downarrow}} \beta_{q,-}^{\dagger}|0\rangle .
$$

Remarkably for $U / t \rightarrow \infty$ and half-filling, by construction Eq. (12) becomes the Néel state with spin quantization parallel to the $x$ axis, i.e. it has the correct Marshall sign on each of the $2^{L}$ configurations sampled by GFMC. In this limit, it is also clear why the Jastrow factor may be much more effective: being defined along the $z$ axis, it allows us to sample the quantum fluctuation perpendicular to the staggered magnetization. In the previous case instead both the Jastrow quantization axis and the order parameter were parallel, and for $U / t \rightarrow \infty$ there is no way to sample any fluctuation, the only possible configuration being the classical one.

It is worth noting that, although for the $t-J$ model the d-wave BCS wave function with a spin-rotationally invariant density-density Jastrow factor represents a very accurate variational state, for the Hubbard model at small and intermediate coupling $(U \leq 10 t)$ the best choice for the variational and guiding wave function is given by a Jastrow-Slater determinant with rotated orbitals Eqs. (10) and (11). Indeed, although the d-wave BCS wave function is a singlet and does not break the $\mathrm{SU}(2)$ symmetry, it has a very poor variational energy for the Hubbard model. The quality of the variational energy obtained with our Jastrow-Slater determinant remains considerably better than the BCS one at half-filling and $U \leq 10 t$ even when the accuracy of the approximation is improved by the GFMC. Instead, in the doped case, the BCS wave function with GFMC is only slightly worse than the corresponding Jastrow-Slater determinant proposed in this work. This may suggest that antiferromagnetism is already suppressed at small finite doping, and $\mathrm{d}$-wave superconductivity is a possible stable phase especially at large $U / t$.

An important systematic improvement of the wave function can be achieved by performing exactly one Lanczos step starting from $\left|\Psi_{G}\right\rangle$,

$$
\left|\Psi_{L}\right\rangle=(1+\alpha H)\left|\Psi_{G}\right\rangle,
$$

with $\alpha$ free parameter chosen to minimize the energy. This technique has been successfully used for the $-J$ model both to improve the variationalcalculation 17 and as a starting point for power methodd18. Henceforth, we will denote by VMC and LS the results obtained with the wave function Eqs. (2) and (13), respectively, using variational Monte Carlo. Analogously, the symbols FN and FNLS will indicate the fixed-node approximation applied to the wave function Eqs. (2) and (13), respectively. Finally, the symbols SR will denote the stochastic reconfiguration approximation applied to the wave function Eq. (13).

In Tables 1 and II we report the energies of 18 and 10 electrons on 18 sites, respectively. At half-filling we compare the results using Eq. (2) with the Slater determinant $|\mathcal{D}\rangle$ given by Eqs. (6) and (12) for different approximations and values of $U / t$. Using Eq. (12) we obtain a sizeable improvement for large $U$ 's $(U \geq 10 t)$. Notice that for $U=20 t$ the best variational result (the FNLS) with Eq. (6) is worse than the simple VMC with Eq. (12). For 10 electrons the two Slater determinants give the same results. Indeed, for this doping the antiferromagnetic order is strongly suppressed and the Jastrow factor $\mathcal{J}$ does not play any important role.

\section{RESULTS}

One of the most debated issues in strongly correlated electron models is the nature of the-harge distribution in their GS. Recently, many author 1925 have addressed the question of PS in the $t-J$ model. It is well accepted that for $J \gg t$, holes tend to cluster together leaving the rest of the system in an antiferromagnetic state without holes. Although most of the calculations lead to the conclusion that a critical value of $J$ below which the GS is homogeneous exists, it is not clear what this value is at low doping, ranging from $0.5 t$ and $1.2 t$. Moreover, some authors 26 have suggested that just before PS, the GS has charge modulations. Recently, an accurate numerical study of a few chains on the Hubbard model27 has shown evidence of stripes, i.e., CDW oscillations. However, this result appears limited to quasi-one-dimensional geometry, as also suggested by the authors.

It is well known that PS is characterized by an infinite compressibility in the thermodynamic limit. The compressibility can be related to the curvature of the energy with respect to the electron density,

$$
\chi=\left(\frac{\partial^{2} E}{\partial n^{2}}\right)^{-1} .
$$

From the above definition we have that a divergent $\chi$ corresponds to a vanishing curvature of the energy as a function of density. Therefore, PS can in principle be detected by means of energy measurements for various densities. This is an appealing property, since the accuracy on the energy is usually better than that of any other observable for most numerical methods. Many previous numerical studies of PS have therefore concentrated on the energy curve, or equivalently, on the energy per hole $e_{h}(\delta)=\left[e(\delta)-e_{H}\right] / \delta$, where $e(\delta)$ is the energy per site at a hole density $\delta=1-n$ and $e_{H}=e(0)$ is the energy at 
half-filling20. If $\chi$ diverges, $e_{h}(\delta)$ is flat in the thermodynamic limit and develops a minimum for $\delta=\delta_{c}$ in finite systems, due to the finite positive surface energy at the phase boundary.

This approach has been pursued by Cosentini et al. 15 , using FN calculations. They found that there is a large region of PS in the phase diagram, at least for $U \geq 10 t$, clearly in contrast with what is found in the $t-J$ model. In this paper, we consider the Hubbard model and we show that a study of PS instability is very difficult using only energy calculation. Instead, a careful calculation of charge-correlation functions strongly indicates that the GS is homogeneous.

In order to show that the energy calculations may overestimate the tendency to a PS instability, it is important to compare the GFMC results with some exact reference results. Previous studies have shown that it is important to consider relatiyely large lattice sizes since finite-size effects favor PS28. We need, therefore, a reference result for large lattices, where exact diagonalization is not available. In the case of the Hubbard model for small $U$, the AFQMC is almost exact and represents the reference we need. As stated in the preceding section, we consider only closed shell doping because, at least for small $U$, huge finite size effects affect the physical properties in a rather drastic way. For instance, the large bare density of states near half-filling determines an unphysical and spurious PS up to the first closed shelled.

In Fig. 1, we show the accuracy of the GFMC results obtained with different approximations compared with the AFQMC ones for a 162-site lattice and $U=4 t$. For this coupling value, AFQMC does not provide evidence for PS. We plot $\left[E(\delta)-E_{h s}(\delta)\right] / E_{h s}(\delta)$, where $E(\delta)$ and $E_{h s}(\delta)$ are the energies of GFMC and AFQMC, respectively, for a doping $\delta$. Besides the improvement in the absolute accuracy, the curves get flatter and flatter improving the approximation, but only the SR accuracy is almost doping-independent. In other words, we need a very accurate calculation to eliminate the spuripus dependence of the variational energy upon dopinges. Even for the best variational method, the FNLS, although the accuracy on the energy is for all doping less than $1 \%$, the difference in accuracy between, for example, the halffilled case and the first closed shell is still sizable. This difference is very important, because it represents just the energy scale determining or ruling out PS.

In Fig. 2 , the function $e_{h}(\delta)$ is shown for the FN, FNLS, $\mathrm{SR}$, and AFQMC methods. We need to use SR to exclude the occurrence of PS, where even the FNLS data would imply PS. The reason for this disappointing situation is that all the known variational methods are still too dependent on the guiding wave function. With the previous analysis, the resolution in energy necessary to detect or rule out PS is very hard to reach with statistical methods, especially for large $U / t$.

On the other hand, GFMC methods have proven to be reliable not only for energy calculations but also for correlation functions such as $N(q)=\left\langle n_{q} n_{-q}\right\rangle$, where $n_{q}$ is the Fourier transform of the electron density24. For a phase-separated system, there are strong fluctuations in the density for small momenta and $N(q \rightarrow 0)$ is expected to be strongly enhanced for small momenta, that is, for $|q| \sim \frac{2 \pi}{\xi}$, where $\xi$ is the characteristic length of the phaseseparated region. Moreover, if $\chi$ diverges, also $N(q \rightarrow 0)$ diverges, yielding an alternative tool to probe PS.

This method turns out to be more reliable, since it is based on a single calculation for a given doping value, whereas the evaluation using $e_{h}(\delta)$ involves a comparison between energies obtained by different simulations for different fillings, with the corresponding guiding wave function having different accuracies. Indeed with GFMC, $N(q)$ has been proved to be a very sensitive tool to look at for detecting PS. In the $t-J$ model, $N(q)$ has a very different shape for stable and unstable systems. A clear peak at the smallest $q$ indicates PS even when, as shown in the inset of Fig. 3, $\mathrm{J} / t$ is very close to the PS boundary 24. Moreover, from $N(q)$ it is also possible to extract information about charge fluctuations at finite $q$ 's, related to CDW. In Ref.24 we have shown that in the $t-J$ model for $J=0.4 t, N(q)$ has some peaks at finite $q$ 's, surprisingly near to what was found in recent experiments 30 . The knowledge of $N(q)$ allows us to extract more general results with respect to the simple study of $e_{h}(\delta)$. Furthermore, $N(q)$ is found to be much less size dependent than $e_{h}(\delta)$. For the $t-J$ model there is no appreciable difference between 98 -site and 162-site lattices and for $J=0.4 t$. Both calculations suggest that there is no PS, whereas an analysis using the FN approximation of $e_{h}(\delta)$ should lead to PS for 98 sites and to a homogeneous state for 162 sites. Indeed, in this case PS is only a size effect and a homogeneous state is found by increasing the accuracy of the method or by increasing the lattice size.

We computed $N(q)$ by means of the forward-walking technique 1 , within the FNLS approximation, at halffilling and for the first few closed-shell configurations on a 162- and a 98-site lattice. The evaluation of the densitydensity correlation function is in principle possible even within SR by numerical differentiation of the energy with respect to an external field coupled to $N(q)$. However, this approach is very demanding and does not give a significant improvement on the FNLS results, which are very accurate. Indeed for the smallest $q$ vector for 90 electrons on the 98 -site lattice, i.e., $q=(2 \pi / 7,2 \pi / 7)$, we found the the AFQMC gives $N(q)=0.0932(2)$ and the FNLS gives $N(q)=0.096(1)$.

In Fig. 3, $N(q)$ is shown for $U=4 t$ at half-filling for a 162-site and a 98 -site lattice and for 154 electrons on a 162-site lattice and 90 electrons on a 98 -site lattice. No sign of divergence, and consequently of PS or CDW, is seen in the data. We also notice that the two sets of points for the half-filled systems lie on the same curve, showing that we have substantially reached the thermodynamic limit. For this value of $U, N(q)$ is essentially featureless for all doping we considered, suggesting that 
there are no charge instabilities at any finite length. The smallest doping we considered is $\delta \simeq 0.049$ and we cannot exclude that for smaller doping PS or CDW are present.

In order to investigate smaller doping, we should consider larger lattices. Unfortunately, the accuracy of the approximations considered decreases when increasing the size of the system and the 162-site lattice represents the largest lattice where the accuracy is acceptable. In Fig. 4, we report $\left[E(0)-E_{h s}(0)\right] / E_{h s}(0)$ for various sizes and for different approximations: from the 18 sites to the 162 sites, the accuracy of FNLS changes from less than $0.1 \%$ to about $0.5 \%$. These indications prevent us from considering sizes larger than the ones presented in this paper.

Now we turn to larger Coulomb interactions and consider $U=10 t$, where the AFQMC results are not reliable due to large fluctuations. In principle, GFMC techniques do not suffer from intrinsic limitations in the large-coupling regime and it is possible to consider any value of $U$. In practice we need an accurate knowledge of the nodes, i.e., an accurate guiding wave function. Our choice, Eq. (2), with orbitals given by Eqs. (10) and (11) is a very good approximation for the half-filling case. In Table If, we report the energies for various methods for 18 electrons on 18 sites at different $U$ 's. Although all the approximations are quite size-dependent, the wave function becomes more and more accurate by increasing the Coulomb potential. Therefore, we expect that it also gives a good starting point at least close to half-filling.

We present results for $U=10 t$, for which previous FN calculations based on $e_{h}(\delta)$ and a less accurate wave function 15 have shown $\mathrm{PS}$ up to $\delta \simeq 0.15$. Indeed, if we use $e_{h}(\delta)$ as a probe for PS, we find that the phase diagram shows a large instability region, confirming the results of Ref.15. As for the $U=4 t$ case, this instability is very likely to be a spurious effect, a consequence of the different energy accuracy for different doping. This possibility, which cannot be proved without knowing the exact energies at strong coupling (at present impossible), is instead very clearly supported by the calculation of the charge-correlation functions.

Figure 5 displays $N(q)$ for the same fillings of Fig. 3 and for 138 electrons on the 162-site lattice, which corresponds to $\delta \simeq 0.148$. All the correlation functions are definitely nondivergent for $q \rightarrow 0$ and are qualitatively similar to the $U=4 t$ case, indicating that the system is far away from a PS instability. Furthermore $N(q)$ does not show peaks at any finite momenta for this Coulomb interaction. This finding shows that the Hubbard and the $t-J$ model may have different behaviors as far as charge correlations are concerned. Indeed, by diagonalizing exactly the 18-site lattice, we find that charge fluctuations for the Hubbard and for the $t-J$ model are quite different in the spall doping region for $U=10 t$ and $J=0.4 t$, respectively 31 .

\section{CONCLUSIONS}

An extensive GFMC analysis of the Hubbard model at low hole doping has been carried out. In particular, we have focused on the possible instability of the model with respect to PS and CDW. Comparing GFMC results with AFQMC ones in the weak-coupling region $(U=4 t)$, we show that detecting PS by means of energy results requires a very accurate calculation at all electronic densities. Indeed, the accuracy of the energy is strongly dependent on the electron density, and the signature of PS based only on energy calculations is clearly affected by this bias, leading to a spurious region of PS instability. In the case of the Hubbard model, this is particularly relevant because, while we are able to give a very good description of the half-filled case, in which the GS is an antiferromagnetic insulator, we are not aware of equally accurate descriptions of the doped state. Even for $U=4 t$, it is necessary to use the really accurate SR and AFQMC technique to eliminate the doping dependence of the accuracy and to rule out PS.

On the other hand, PS (and CDW) instability can be probed more easily using charge correlation functions. This approach has various advantages. First, it is found that $N(q)$ has very small size effects and the thermodynamic limit is reached with about 100 sites, both for the Hubbard and the $t-J$ model. Second, the information contained in $N(q)$ does not depend on different densities, implying that a different accuracy as a function of doping does not introduce any external bias.

Instead of using energy calculations, which are very expensive at moderate and large U's, we calculate the charge correlation functions and we are able to find clear evidence for the absence of PS up to $U=10 t$ in the low doping regime.

\section{ACKNOWLEDGMENTS}

It is a pleasure to acknowledge useful discussions with L. Capriotti, M. Calandra, G. Bachelet, E. Koch, and A. Parola, to whom we are also grateful for critical and careful reading of the manuscript. This work has been partially supported by MURST-COFIN99 and by Istituto Nazionale per la Fisica della Materia. 


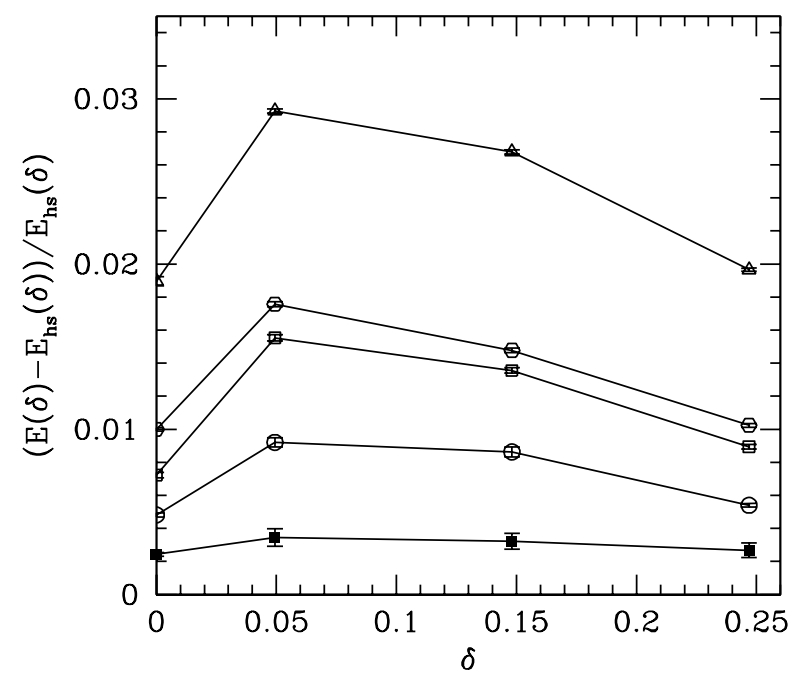

FIG. 1. Relative accuracy of various GFMC techniques with respect to AFQMC for a 162-site lattice with $U=4 t$ as a function of filling $\delta$. From top to bottom VMC (empty triangles), FN (empty hexagons), LS (empty squares), FNLS (empty circles), and SR (full squares). Lines are guides to the eye.

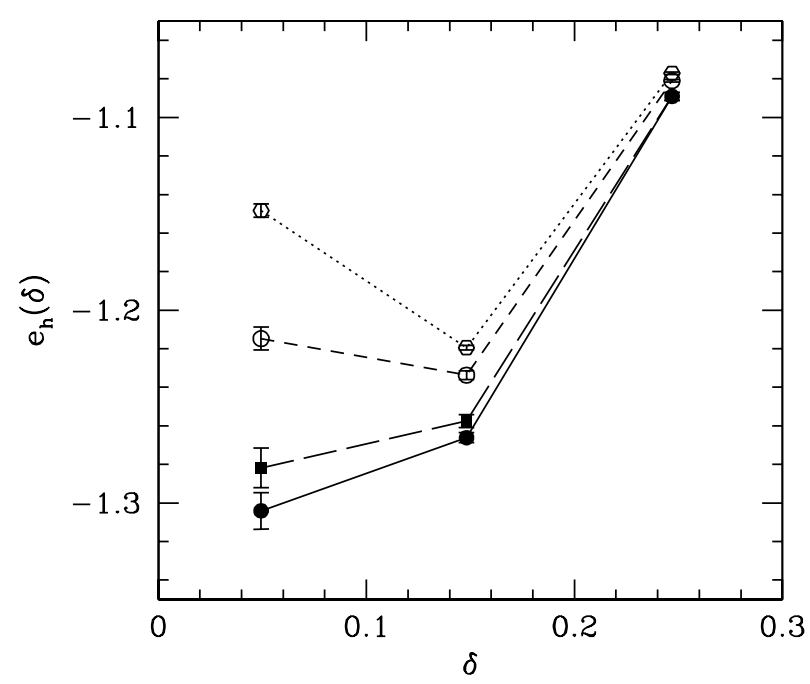

FIG. 2. Energy per hole $e_{h}(\delta)$ for a 162-site lattice with $U=4 t$. From top to bottom FN (empty hexagons), FNLS (empty circles), SR (full squares), and AFQMC (full circles). Lines are guides to the eye.

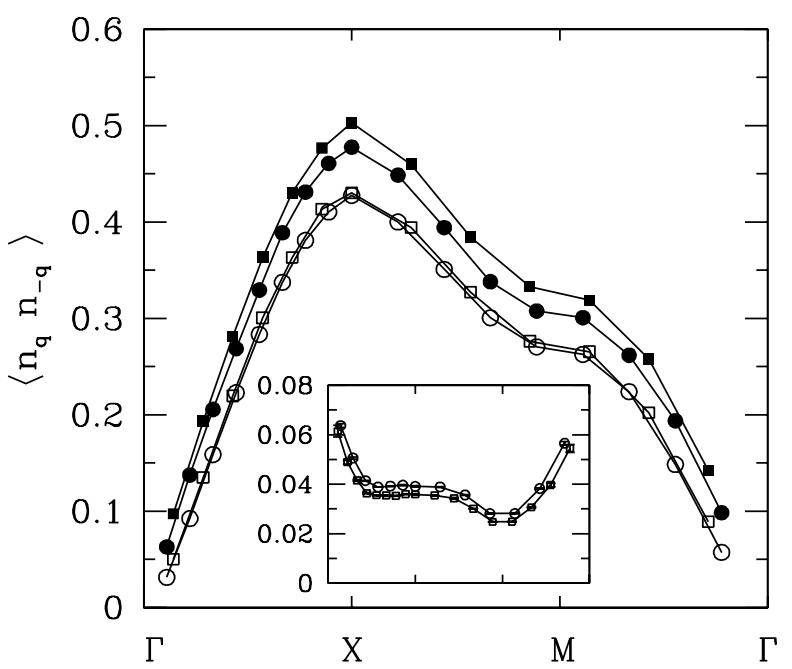

FIG. 3. $N(q)$ for $U=4 t, 162$ electrons on 162 sites (empty circles), 98 electron on 98 sites (empty squares), 154 electrons on 162 sites (full circles) and 90 electrons on 98 sites (full squares). Lines are guides to the eye and error bars are smaller than points. $\Gamma=(0,0), X=(\pi, \pi), M=(\pi, 0)$. In the inset: $N(q)$ for the $t-J$ model, $J=0.6 t, 156$ electrons on 162 sites (squares), and 94 electrons on 98 sites (circles).

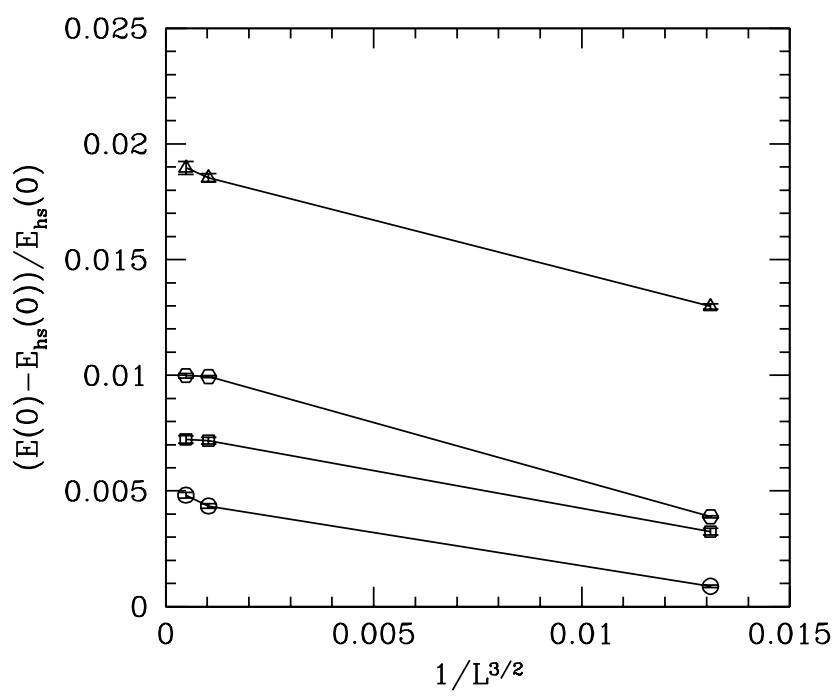

FIG. 4. Relative accuracy of various GFMC techniques with respect to AFQMC for different lattices $(L=18,98,162)$ and $U=4 t$. From top to bottom: VMC (empty triangles), FN (empty hexagons), LS (empty squares), and FNLS (empty circles). Lines are guides to the eye. 


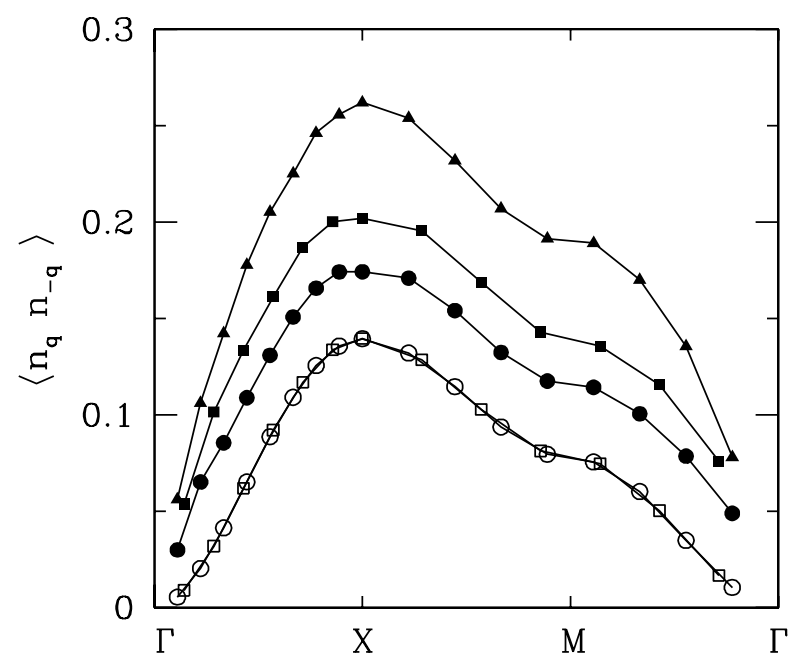

FIG. 5. $\quad N(q)$ for $U=10 t, 162$ electrons on 162 sites (empty circles), 98 electrons on 98 sites (empty squares), 154 electrons on 162 sites (full circles), 90 electrons on 98 sites (full squares), and 138 electrons on 162 sites (full triangles). Lines are guides to the eye and error bars are smaller than points. $\Gamma=(0,0), X=(\pi, \pi), M=(\pi, 0)$.

\begin{tabular}{lllllll}
\hline \hline$U$ & $|\mathcal{D}\rangle$ & $E_{e x}$ & $E_{V M C}$ & $E_{F N}$ & $E_{L S}$ & $E_{F N L S}$ \\
\hline \hline $4 \mathrm{t}$ & $(6)$ & -0.9585 & $-0.9382(1)$ & $-0.9514(1)$ & $-0.9520(1)$ & $-0.9556(1)$ \\
$10 \mathrm{t}$ & 6 & -0.4484 & $-0.4034(1)$ & $-0.4284(1)$ & $-0.4154(1)$ & $-0.4316(1)$ \\
$20 \mathrm{t}$ & 6 & -0.2339 & $-0.2023(1)$ & $-0.2195(1)$ & $-0.2060(1)$ & $-0.2225(1)$ \\
$4 \mathrm{t}$ & $(12)$ & -0.9585 & $-0.9460(1)$ & $-0.9547(1)$ & $-0.9553(1)$ & $-0.9576(1)$ \\
$10 \mathrm{t}$ & 12 & -0.4484 & $-0.4382(1)$ & $-0.4451(1)$ & $-0.4428(1)$ & $-0.4470(1)$ \\
$20 \mathrm{t}$ & $(12)$ & -0.2339 & $-0.2293(1)$ & $-0.2232(1)$ & $-0.2310(1)$ & $-0.2337(1)$ \\
\hline \hline
\end{tabular}

TABLE I. GS energies for 18 electrons on 18 sites as a function of $U / t$ using (6) and (12) as Slater determinant.

\begin{tabular}{llllll}
\hline \hline$U$ & $E_{e x}$ & $E_{V M C}$ & $E_{F N}$ & $E_{L S}$ & $E_{F N L S}$ \\
\hline \hline $4 \mathrm{t}$ & -1.1299 & $-1.1124(1)$ & $-1.1218(1)$ & $-1.1229(1)$ & $-1.1263(1)$ \\
$10 \mathrm{t}$ & -1.0193 & $-0.9749(1)$ & $-1.0006(1)$ & $-0.9997(1)$ & $-1.0098(1)$ \\
$20 \mathrm{t}$ & -0.9598 & $-0.8983(1)$ & $-0.9354(1)$ & $-0.9253(1)$ & $-0.9450(1)$ \\
\hline \hline
\end{tabular}

TABLE II. GS energies for 10 electrons on 18 sites as a function of $U / t$.
${ }^{1}$ J. Hubbard, Proc. R. Soc. London, Ser. A 276, 238 (1963); M.C. Gutzwiller, Phys. Rev. Lett. 10, 159 (1963); J. Kanamori, Prog. Theor. Phys. 30, 275 (1963).

2 J.D. Jorgensen, B. Dabrowski, Shiyon Pei, D.G. Hinks, L. Soderholm, B. Morosin, J.E. Shirber, E.L. Venturini, and D.S. Ginley, Phys. Rev. B 38, 11337 (1988).

3 J.M. Tranquada, B.J. Sternlib, J.D. Axe, Y. Nakamura, and S. Uchida, Nature 375, 561 (1995).

${ }^{4}$ V.J. Emery and S.A. Kivelson, Physica C 209, 597 (1993).

${ }^{5}$ C. Castellani, C. Di Castro, and M. Grilli, Phys. Rev. Lett. 75, 4650 (1995).

${ }^{6}$ S.R. White, Phys. Rev. Lett. 69, 2863 (1992); S.R. White, Phys. Rev. B 48, 10345 (1993).

${ }^{7}$ D.R. Hamann and S.B. Fahy, Phys. Rev. B 41, 11352 (1990); S.B. Fahy and D.R. Hamann, Phys. Rev. Lett. 65, 3437 (1990).

${ }^{8}$ N. Trivedi and D.M. Ceperley, Phys. Rev. B 41, 4552 (1990).

9 Shiwei Zhang, J. Carlson, and J.E. Gubernatis, Phys. Rev. Lett. 74, 3652 (1995); Shiwei Zhang, J. Carlson, and J.E. Gubernatis, Phys. Rev. B 55, 7464 (1997).

${ }^{10}$ D.F.B. ten Haaf, H.J.M. van Bemmel, J.M.J. van Leeuwen, W. van Saarloos, and D.M. Ceperley, Phys. Rev. B 51, 13039 (1995)

${ }^{11}$ S. Sorella, Phys. Rev. Lett. 80, 4558 (1998).

12 S. Sorella and L. Capriotti, Phys. Rev. B 61, 2599 (2000).

${ }^{13}$ F. Franjic and S. Sorella, Prog. Theor. Phys. 97, 399 (1997).

${ }^{14}$ H. Yokoyama and H. Shiba, J. of Phys. Soc. of Japan. 56, 3582 (1987).

15 A. Cosentini, M. Capone, L. Guidoni, and G. Bachelet, Phys. Rev. B 58, 14685 (1998).

${ }^{16}$ M. Calandra and S. Sorella, Phys. Rev. B 57, 11446 (1998).

17 E.S. Heeb and T.M. Rice, Europhys. Lett. 27, 673 (1994).

18 Y.C. Chen and T.K. Lee, Phys. Rev. B 51, 6723 (1995).

${ }^{19}$ C.T. Shih, Y.C. Chen, and T.K. Lee, Phys. Rev. B 57, 627 (1998).

${ }^{20}$ V.J. Emery, S.A. Kivelson, and H.Q. Lin, Phys. Rev. Lett. 64, 475 (1990).

${ }^{21}$ W.O. Putikka, M.U. Lucchini, and T.M. Rice, Phys. Rev. Lett. 68, 538 (1992).

${ }^{22}$ C.S. Hellberg and E. Manousakis, Phys. Rev. Lett. 78, 4609 (1997).

${ }^{23}$ M. Kohno, Phys. Rev. B 55, 1435 (1997).

${ }^{24}$ M. Calandra, F. Becca, and S. Sorella, Phys. Rev. Lett. 81, 5185 (1998).

${ }^{25}$ S. Rommer, S.R. White, and D.J. Scalapino, Phys. Rev. B 61, 13424 (2000).

${ }^{26}$ S.R. White and D.J. Scalapino, Phys. Rev. Lett. 80, 1272 (1998); 81, 3227 (1998).

27 J. Bonca, J.E. Gubernatis, M. Guerrero, E. Jeckelmann, and S.R. White, Phys. Rev. B 61, 3251 (2000).

${ }^{28}$ N. Furukawa and M. Imada, J. of Phys. Soc. of Japan 61, 3331 (1992).

${ }^{29}$ We checked that without rotating orbitals, that is, using 
the wave function of Ref. 5 , we have much less accuracy as a function of density.

${ }^{30}$ J.M. Tranquada, J.D. Axe, N. Ichikawa, A.R. Moodenbaugh, Y. Nakamura, and S. Ucida, Phys. Rev. Lett. 78, 338 (1997).

${ }^{31}$ F. Becca, A. Parola, and S. Sorella, Phys. Rev. B 61, 16287 (2000). 